\title{
CORRECTION
}

\section{Correction to: Building heterogeneous ensembles by pooling homogeneous ensembles}

\author{
Maryam Sabzevari ${ }^{1} \cdot$ Gonzalo Martínez-Muñoz ${ }^{2} \cdot$ Alberto Suárez $^{2}$
}

Published online: 17 December 2021

๑) Springer-Verlag GmbH Germany, part of Springer Nature 2021

\section{Correction to: \\ International Journal of Machine Learning and \\ Cybernetics \\ https://doi.org/10.1007/s13042-021-01442-1}

Unfortunately, the article has been published without acknowledgment. The correct acknowledgment is given below.

The authors acknowledge financial support from PID2019-106827GB-I00/AEI/10.13039/501100011033.

Publisher's Note Springer Nature remains neutral with regard to jurisdictional claims in published maps and institutional affiliations.

The original article can be found online at https://doi.org/10.1007/ s13042-021-01442-1.

Maryam Sabzevari

maryam.sabzevari@aalto.fi

1 Computer Science Department, Aalto University,

Konemiehentie 2, 02150 Espoo, Finland

2 Escuela Politécnica Superior, Universidad Autónoma de Madrid, Ciudad Universitaria de Cantoblanco, 28049 Madrid, Spain 